\title{
Progesterone regulation of uterine dendritic cell function in rodents is dependent on the stage of estrous cycle
}

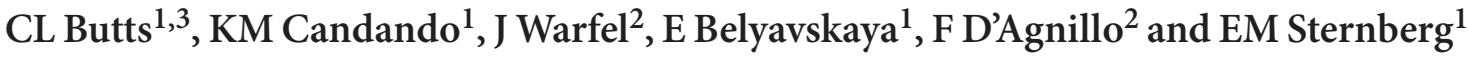

Steroid hormones, such as progesterone, are able to modify immunity and influence disease outcome. Dendritic cells (DCs) drive potent immune responses, express receptors for steroid hormones, and may be a primary target of steroid hormone actions during infection of the genital tract, including uterine tissue. Here, we report that progesterone limited DC-associated activation marker expression and inhibited cytokine secretion by uterine DCs, which was associated with changes in signal transducer and activator of transcription 1 (STAT1) activity. We also found that DCs from mice at stages with higher progesterone concentrations (diestrus, metaestrus) were more sensitive to progesterone than those in stages with lower progesterone concentrations (proestrus, estrus), both in vitro and in vivo. This difference correlated with the levels of progesterone receptor expressed by DCs. These data suggest that progesterone regulates DC function and could contribute to the susceptibility of females to uterine and other genital tract infections at selected time periods throughout the life cycle.

\section{INTRODUCTION}

Women exhibit increased susceptibility to a number of genital tract infectious agents compared with men (human immunodeficiency virus (HIV), ${ }^{1}$ genital herpes, ${ }^{2}$ Chlamydia. $\left.{ }^{3,4}\right)$. One factor that could account for this difference is variation in steroid hormone levels. Steroid hormones fluctuate throughout the menstrual cycle, can modify immunity, and contribute to changes in immune responses. This could facilitate infections in the female genital tract ${ }^{5-7}$ that are exposed to and influenced by the sex hormones estrogen and progesterone. Defining the mechanism by which each of these hormones regulates immunity is important for determining their relative contribution to susceptibility to infectious agents.

Female genital tract (uterus, cervix, and vagina) mucosal tissues are regulated by several factors commonly produced in the mucosa, as well as by estrogen and progesterone. ${ }^{8}$ Immune cells, such as dendritic cells (DCs), in the female genital tract differ from those in other mucosal sites because of the microenvironment and their potential exposure to the exterior environment. They must traverse the multilayer squamous epithelium of genital tract tissues for antigen uptake and trafficking to regional lymph nodes to induce potent immune responses against infectious agents. ${ }^{9,10} \mathrm{~A}$ recent study reported that macrophages from vaginal tissue allow HIV-1 replication, whereas those from the intestinal lamina propria do not, ${ }^{11}$ which emphasizes the concept that factors in the genital tract alter immune cell function in a manner different from other mucosal sites.

Dendritic cells are considered crucial in ameliorating a number of diseases, ${ }^{12-14}$ and can initiate strong responses against pathogens. ${ }^{15,16}$ In the genital tract, they are able to extend their processes between epithelial cells and may be involved in pathogen entry. ${ }^{9}$ Immature DCs efficiently process antigenic peptides and are thought to be important in initiating tolerogenic responses, ${ }^{17,18}$ Mature DCs (stimulated by activating agents such as the bacterial cell wall component lipopolysaccharide-LPS) present antigen in the context of major histocompatibility complex (MHC) molecules and secrete cytokines (tumor necrosis factor- $\alpha$-TNF- $\alpha$, interleukin $1 \beta$-IL- $1 \beta$ ) that promote inflammatory immune responses. ${ }^{19,20} \mathrm{DC}$ maturation can be induced by a number of different mechanisms, such as the Janus kinase (JAK), signal transducer and activator of transcription (STAT) signaling 
pathway. ${ }^{21}$ This includes STAT1 proteins, which form dimers that bind to target genes and modify cellular activity. ${ }^{22}$ In addition, loss of STAT1 function has been shown to greatly reduce innate resistance to intracellular pathogens. ${ }^{23}$ Mature DCs also express high levels of MHC-peptide complexes and costimulatory molecules, such as CD80 and CD86, on their cell surface. Expression of these molecules and secretion of cytokines such as IL-12 allow DCs to stimulate naive T lymphocytes ${ }^{24}$ and direct potent helper $\mathrm{T}$ cell $\left(\mathrm{T}_{\mathrm{H}} 1, \mathrm{~T}_{\mathrm{H}} 2, \mathrm{~T}_{\mathrm{H}} 17\right.$, Treg) responses. ${ }^{25-27}$ Several DC subsets have been identified, primarily on the basis of cell surface molecules expression. ${ }^{28} \mathrm{CD} 11 \mathrm{c}^{+} \mathrm{DCs}$ are among the most abundant and have been identified in both lymphoid (spleen) $)^{25,29}$ and nonlymphoid (uterus) $)^{9,30,31}$ tissues.

The ovarian steroid hormone progesterone, whose levels fluctuate throughout the menstrual cycle, is critical for ovulation $^{32}$ and uterine cell function, ${ }^{33}$ especially during pregnancy. ${ }^{34}$ Its actions are typically mediated by binding to progesterone receptors (PRs) in the cytoplasm of cells, which dimerize and translocate to the nucleus to modify expression of PR-specific target genes. ${ }^{35}$ Several studies have reported immunomodulatory effects of progesterone, ${ }^{36,37}$ including inhibition of cytotoxic T- and NK-cell activity, ${ }^{38}$ decreased production of IgG and IgA antibodies, ${ }^{2}$ and changes in lymphocyte migration and proliferation. ${ }^{39}$ Progesterone has been shown to increase the number of Langerhans cells (skin DCs) in human vaginal epithelium, ${ }^{40}$ and the use of progesterone-based contraceptives is associated with increased risk of HIV-1 infection and higher viral loads as well as accelerated disease progression and mortality. ${ }^{41}$ It is also reported to have direct effects on $\mathrm{T}$ lymphocytes at pregnancy concentrations, suggesting a role in preventing maternal immune responses against fetal antigens. ${ }^{38}$ Estrogen, another steroid hormone produced during the menstrual cycle, has been extensively studied and shown to have both immunostimulatory ${ }^{42-44}$ and immunosuppressive effects ${ }^{45-47}$ on DCs ${ }^{48,49}$ and other immune cells, depending on its concentration.

Our group previously showed that, although effects on immature DCs were less dramatic, progesterone treatment of mature bone marrow-derived DCs (BMDCs) from rats led to inhibition of proinflammatory cytokine (TNF- $\alpha$, IL- $1 \beta$ ) secretion, limited expression of DC-associated activation markers (MHC class II, CD80), and a reduced capacity to stimulate proliferation of $\mathrm{T}$ lymphocytes, ${ }^{50}$ which was reversed by the PR antagonist RU486. Other groups have similarly reported modulation of DC function by progesterone, including classical (monocyte-derived) ${ }^{51}$ and plasmacytoid DCs. ${ }^{52}$

In this study, we further explored progesterone effects on DC function in mice. Progesterone has been reported to interfere with STAT1 activity in epithelial cells ${ }^{53}$ and could modify DC function by altering STAT1 expression. We show here that progesterone treatment inhibited STAT1 activity in these cells. We also examined progesterone effects on uterine DC function at different stages of the estrous cycle, and show that the immune-modulating effects of progesterone vary depending on estrous cycle stage and that this is likely due to differences in PR expression by these cells. Differential in vivo responsiveness we show here indicates that progesterone has a physiological regulatory role on DC-mediated immune responses which has important implications for differences in female susceptibility to immune-mediated and infectious diseases across the menstrual and life cycle.

\section{RESULTS}

\section{Progesterone inhibits murine DC activation}

We previously showed that progesterone upregulated PR expression but limited MHC class II and costimulatory molecule (CD80) expression by mature BMDCs from rats in a dose-dependent manner. ${ }^{50}$ To further explore the role of progesterone and its effects on DCs in tissues in which progesterone is known to regulate cellular activity (female genitourinary tract), we used a mouse model that is more suitable for immunological assessment of mucosal tissues. ${ }^{54}$ Splenic DCs were pretreated with progesterone at varying concentrations (consistent with females in a nonpregnant state) and analyzed for expression of the murine MHC class II molecule I- $\mathrm{A}^{\mathrm{b}}$ and costimulatory molecule CD80 (Figure 1a). Consistent with our previous report using rat BMDCs, LPS stimulation upregulated expression of CD80 and I- $\mathrm{A}^{\mathrm{b}}$ by splenic DCs. Progesterone pretreatment of LPS-activated splenic DCs limited I- $\mathrm{A}^{\mathrm{b}}$ expression and, more dramatically, CD80 expression by these cells. Other cell surface molecules associated with DC activation (Toll-like receptor 4-TLR4, CD86, and CD40) were examined, and progesterone also limited expression of these molecules by mature splenic DCs (data not shown).

To evaluate progesterone effects on cytokine production by murine DCs and compare with previous studies using rat BMDCs, proinflammatory cytokine secretion by splenic DCs was measured. Our previous study showed that progesterone suppressed proinflammatory cytokine secretion (TNF- $\alpha$, IL-1 $\beta$ ), but did not alter IL-10 production by rat BMDCs. As shown in Figure $\mathbf{1 b}, \mathbf{c}$ (similar to rat BMDCs), progesterone inhibited mature splenic DC secretion of the proinflammatory cytokines TNF- $\alpha$ and IL-1 $\beta$ in a dosedependent manner. We also found that progesterone inhibited IL-12 secretion, a cytokine known to promote $\mathrm{T}_{\mathrm{H}} 1$ responses (Figure 1d). To determine whether this effect was mediated through the PR, cells treated with progesterone were cocultured with the PR antagonist RU486. RU486 prevented progesterone-mediated suppression of TNF- $\alpha$, IL-1 $\beta$, and IL-12 secretion (Figure 1), indicating that progesterone inhibition of cytokine production by mature murine splenic DCs is likely mediated through its receptor. Progesterone inhibition of DC-mediated T-cell proliferation were previously reported with rat BMDCs and also identified with splenic DCs from mice (unpublished results). These data show similar responses to progesterone by murine and rat DCs.

\section{Progesterone inhibits STAT1 activity}

Signal transducer and activator of transcription 1 (STAT) is a transcription factor that is known to control cytokine production in DCs and other immune cells, ${ }^{23,55}$ and is activated by tyrosine phosphorylation. ${ }^{22}$ Progesterone has been shown to interfere with STAT1 activity in epithelial cells ${ }^{53,56,57}$ and, therefore, could alter STAT1 activity in DCs to modify cellular function. STAT $1 \alpha$ and phosphorylated STAT $1 \alpha / \beta$ (pSTAT1) expression were measured after pretreatment with progesterone and LPS stimulation. 
a

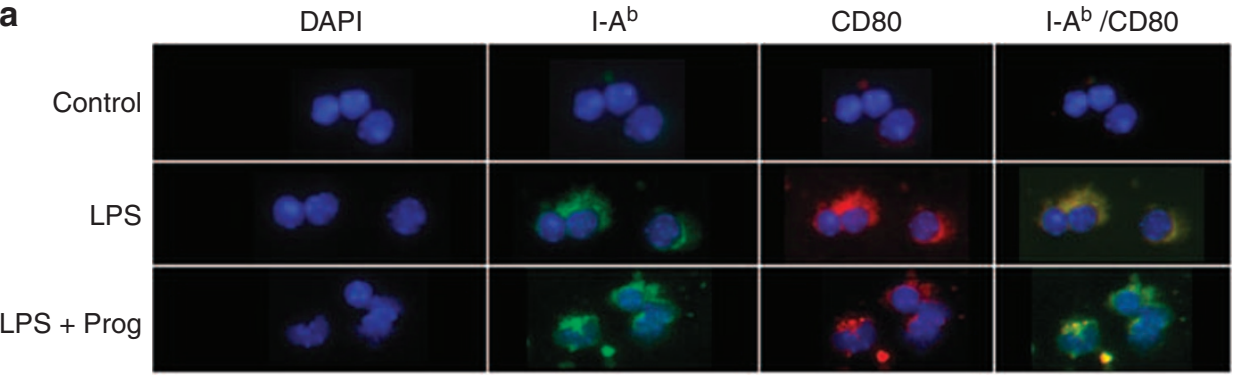

b

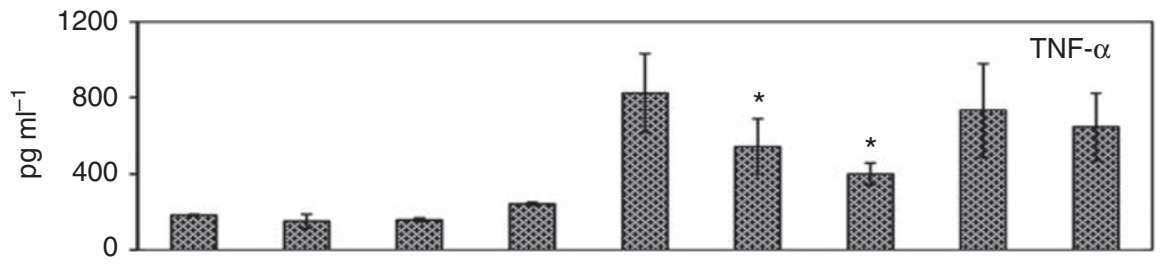

C

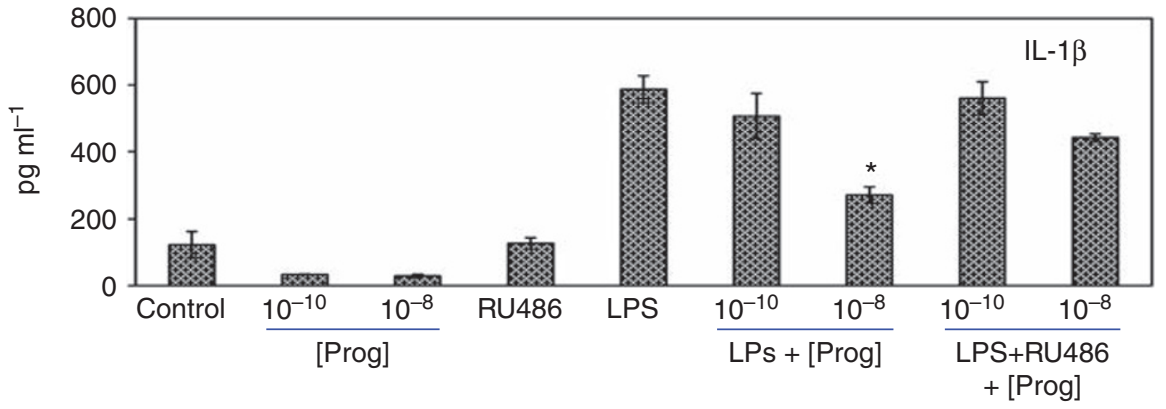

d

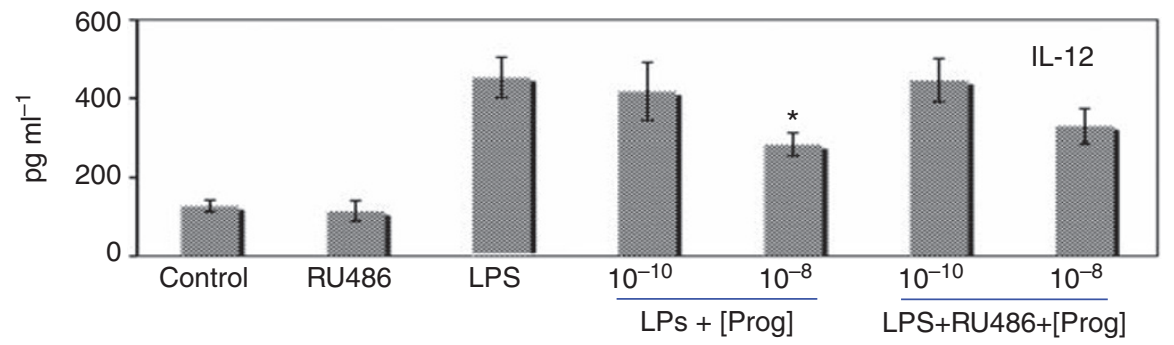

Figure 1 Progesterone effects on murine dendritic cell (DC) function. (a) Fluorescent micrographs show expression of costimulatory molecule CD80 and major histocompatibility complex class II molecule I-A $\mathrm{A}^{\mathrm{b}}$ by splenic DCs after pretreatment with progesterone and lipopolysaccharide (LPS) activation. Micrographs representative of five independent experiments. Tumor necrosis factor- $\alpha-\mathrm{TNF}-\alpha(\mathbf{b})$, interleukin $1-\beta-\mathrm{IL}-1 \beta$ (c), and interleukin 12-IL-12 (d) secreted by LPS-stimulated splenic DC was measured. Progesterone inhibits secretion of TNF- $\alpha$, IL-1 $1 \beta$, and IL-12 cytokines by cultured splenic DCs in a dose-dependent manner, and this is prevented by the progesterone receptor antagonist RU486. Mean \pm s.d. ${ }^{*}=P<0.05$ ( $n=9$ mice).

STAT $1 \alpha$ expression in splenocytes did not dramatically change after treatment with progesterone, LPS, or a combination of both (Figure 2a). LPS induced an increase in pSTAT1 expression, and progesterone reduced pSTAT1 expression in splenocytes in the presence or absence of LPS (pSTAT1/STAT1 ratio: control-1; LPS - 2.07; progesterone-0.57; LPS + progesterone-1.48; IFN$\gamma-17.3$ ) (Figure 2b). This suggests that progesterone is able to modify STAT1 activation in immune cells that express this transcription factor, including DCs.

\section{Progesterone effects on splenic DCs throughout the estrous cycle}

Sex hormone levels fluctuate during the menstrual cycle of women and estrous cycle of female mice (Figure 3a). Therefore, examining progesterone effects on DCs at different stages of the estrous cycle will help establish whether there are specific stages of the estrous cycle in which progesterone has a more prominent role in regulating immune cell activity. Vaginal smears were collected from female mice over a 30-day period to develop a system for delineating each stage of the estrous cycle (Figure $3 \mathbf{b}$ ), The vaginal tract of mice in proestrus is characterized by predominance of round, nucleated vaginal epithelium (ovoid cells); estrus primarily consists of large irregularshaped, anucleated cornified epithelial cells; metaestrus contains mostly infiltrating leukocytes and is identified by the presence of cornified vaginal epithelial cells and polymorphonuclear cells (leukocytes); and diestrus primarily consists of leukocytes and some epithelial cells (nucleated, much larger than leukocytes). After establishing a system for determining each stage of the estrous cycle, immature splenic DCs were isolated from 

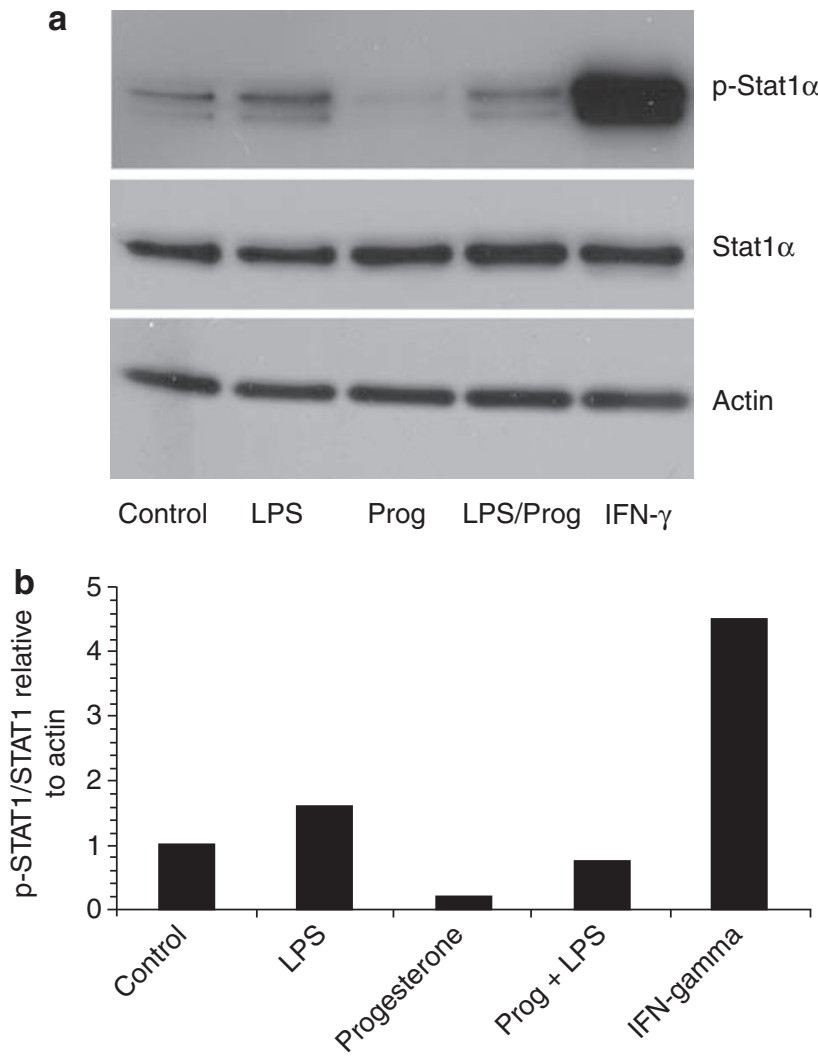

Figure 2 Effects of progesterone on signal transducer and activator of transcription 1 (STAT1) activity. Splenocytes were pretreated with progesterone for $2 \mathrm{~h}$ and then stimulated with lipopolysaccharide (LPS). pSTAT $1 \alpha$ and STAT $1 \alpha$ expression by splenocytes was measured was measured 20 min after culturing using western blot analysis (a). (b) Graph shows pSTAT1/STAT1 values relative to actin and normalized to the control (untreated cells) as determined by densitometry.

individual female mice, analyzed for expression of PR, and grouped on the basis of specific stage of the estrous cycle. As shown in Figure 3c, a greater proportion of DCs from female mice at stages of the estrous cycle associated with higher progesterone levels (metaestrus, diestrus) expressed PR, whereas a smaller proportion of DCs from mice at stages associated with lower progesterone levels (proestrus, estrus) expressed PR: metaestrus, $71.47 \pm 3.85$; diestrus, $77.73 \pm 2.45$; proestrus, 49.80 \pm 8.75 ; and estrus, $62.64 \pm 7.29$. This would indicate that progesterone potentially has different effects on splenic DCs, depending on stage of the estrous cycle.

To assess whether progesterone has varying effects on splenic DC function throughout the estrous cycle, CD80 expression and TNF- $\alpha$ secretion by LPS-stimulated splenic DCs that were isolated from female mice at different stages of the estrous cycle were evaluated. Splenic DCs from mice in stages of the estrous cycle associated with higher progesterone levels (metaestrus, diestrus) secreted lower levels of TNF- $\alpha$ and fewer proportions of DCs upregulated CD80 expression in response to LPS, compared with DCs from mice in stages of the estrous cycle associated with lower progesterone levels (proestrus, estrus): TNF- $\alpha$ : proestrus $-896.2 \pm 41.7$, estrus $-776.4 \pm 19.26$, metaestrus $-475.5 \pm 31.04$, diestrus $-548.4 \pm 16.90$; CD80: proestrus $-74.2 \pm 6.67$, estrus $-78.0 \pm 7.12$, metaestrus $-64.3 \pm 3.49$, diestrus $-57.3 \pm 3.91$ (Figure 4). Furthermore, splenic DCs from mice in stages of the estrous cycle associated with higher progesterone levels were more sensitive to progesterone inhibition, as shown by a sharper decline in TNF- $\alpha$ secretion and CD80 expression by these cells after progesterone treatment. This suggests that progesterone regulation of splenic DC function depends on the stage of the estrous cycle.

\section{Progesterone effects on uterine DCs at specific estrous cycle stages}

The estrous cycle is a dynamic process, characterized by changes in hormonal levels, vaginal mucosa, ovaries, and the uterus. Uterine tissue morphology at each stage of the estrous cycle was examined, and animals in stages of the estrous cycle associated with high progesterone levels (diestrus) were found to contain a greater number of glandular epithelial cells (Figure 5a). Differences in morphological features could have a key role in access of viruses and other pathogens to the uterine cavity and also the ability of immune cells to traverse the uterine epithelium.

It is possible that progesterone secreted in uterine tissue at varying levels throughout the estrous cycle could differentially modify immune cell activity. Expression of TLR4, the receptor for LPS, by DCs was evaluated (Figure 5b). Higher proportions of splenic and uterine DCs isolated from mice at stages of the estrous cycle associated with lower progesterone levels (estrus), expressed TLR4, which would indicate differences in the ability of DCs at these stages to respond to LPS and TLR4-stimulating pathogens. To evaluate the functional relevance of these differences, cytokine production after LPS stimulation was measured. Uterine DCs from mice at stages of the estrous cycle associated with higher progesterone levels (diestrus) secreted less TNF- $\alpha$ in response to LPS (estrus $-191.10 \pm 3.11$, diestrus $-146.60 \pm 25.60$ ) and were more sensitive to the inhibitory effects of progesterone (Figure 5c). In addition, there were fewer CD11 $\mathrm{c}^{+}$DCs in the uterine tissue obtained from mice in stages of the estrous cycle associated with high progesterone levels (diestrus) (data not shown). These data suggest that progesterone is able to modify splenic and uterine DC function in mice and that the impact of progesterone on DCs in the female genital tract depends on the stage of the estrous cycle.

It is important to assess whether these differences in DC responses occur in vivo to substantiate progesterone limiting DC function during infection of the female genital tract by viruses and other pathogens. A system was developed using intravaginal (IVAG) administration of a fluoroisothiocyanate (FITC)-labeled protein (FITC-dextran) in animals at stages of the estrous cycle stage associated with low (estrus) and high (diestrus) progesterone levels to assess any differences in uterine DC function during infection in vivo (Supplementary Figure 1). Uterine, regional lymph node, and splenic tissue were collected $24 \mathrm{~h}$ after IVAG administration to identify FITC-dextran expression in tissues, which were shown to be primarily in lymph node and splenic 
a
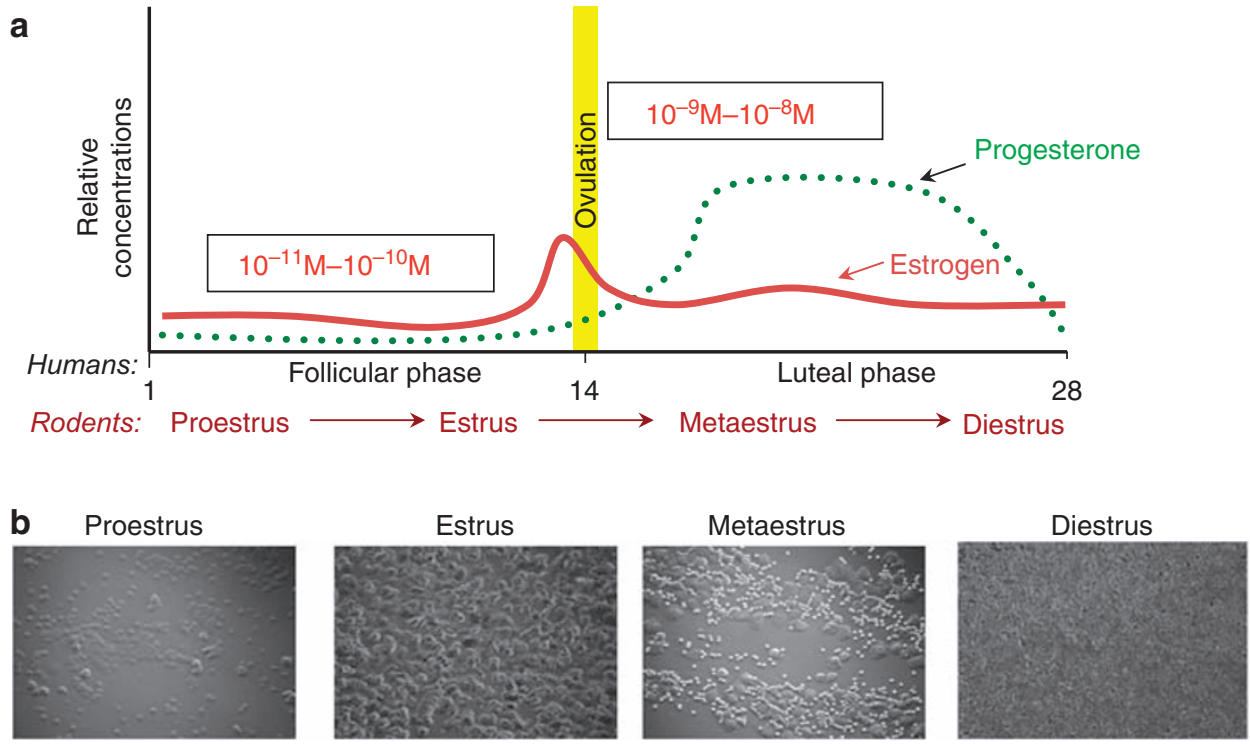

C

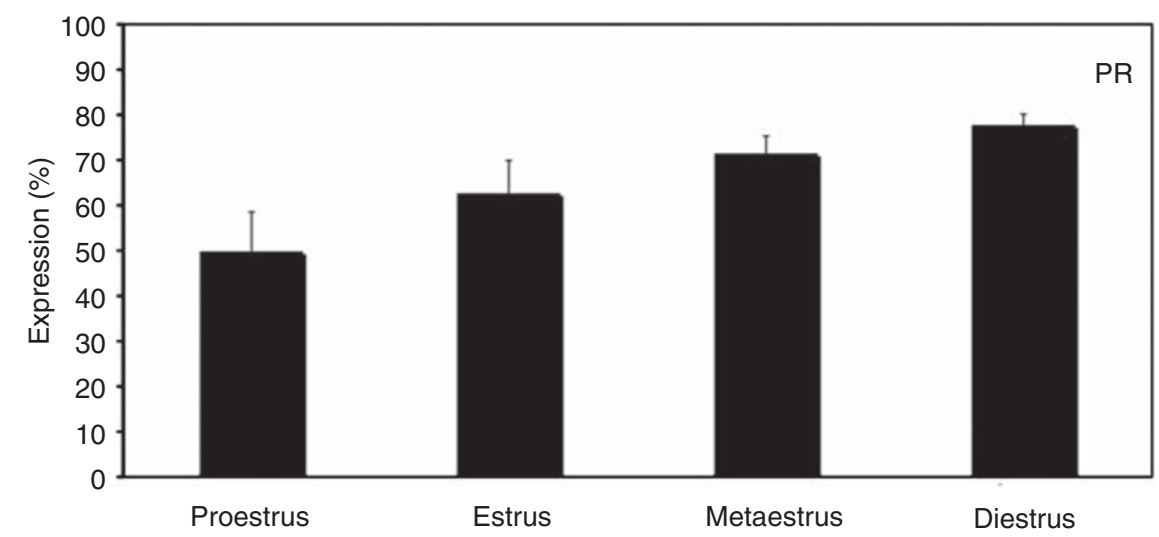

Figure 3 Progesterone levels vary throughout the female cycle. (a) Model shows typical cycle in humans (menstrual) and rodents (estrous) with relative concentrations of progesterone and estrogen during each stage. Mice were separated into specific stages of the estrous cycle, which were determined by vaginal smears as shown in (b). Micrographs representative of six independent experiments, Expression of progesterone receptor (PR) by immature splenic dendritic cells (DCs) was determined (c). Mean \pm s.d. 8 mice per group.

tissues. LPS was administered IVAG to study effects on uterine DC function. High expression of CD80 by splenic and lymph node CD11 $\mathrm{c}^{+}$DCs from mice in estrus after LPS administration was identified, but much less expression by splenic and lymph node CD $11 \mathrm{c}^{+}$DCs from mice in diestrus (Figure 6). This suggests that during infection, murine DC function in the female genital tract may differ throughout the estrous cycle.

\section{DISCUSSION}

In this study, we show that progesterone differentially regulates DC function in the female genital tract throughout the estrous cycle. DCs have an important role in mounting strong host immune responses to pathogens and serve as the interface between innate and adaptive immunity. ${ }^{25,29,58,59}$ Consequently, varying progesterone effects on DC function throughout the estrous cycle could contribute to variations in female susceptibility to infectious diseases of the genital tract by influencing DC-mediated immunity.
We previously explored the role of progesterone on rat DC function and found that it had a greater impact on mature DC function compared with immature DCs. ${ }^{50}$ This could be related to the intracellular properties of immature and mature DCs and what proteins they express. ${ }^{60}$ Here, we sought to further explore progesterone effects on DC function in lymphoid and hormone-responsive mucosal tissues using a model commonly used for immunological studies on mucosal sites (mouse). Our findings indicate that progesterone modifies uterine and splenic DC activity and that this is dependent on expression of PR by these cells. Progesterone decreased DC expression of cell surface markers associated with activation $\left(\mathrm{I}-\mathrm{A}^{\mathrm{b}}, \mathrm{CD} 80\right)$ and inhibited secretion of proinflammatory (TNF- $\alpha$ and IL-1 $\beta$ ) and $\mathrm{T}_{\mathrm{H}} 1$ response-promoting (IL-12) cytokines by DCs. The dose-dependent inhibition of TNF- $\alpha$ production by progesterone is consistent with previous studies showing TNF- $\alpha$ is a target gene of the PR. ${ }^{61}$ Furthermore, the PR antagonist RU486 prevented progesterone-induced inhibition of cytokine production by DCs, signifying the relevance 

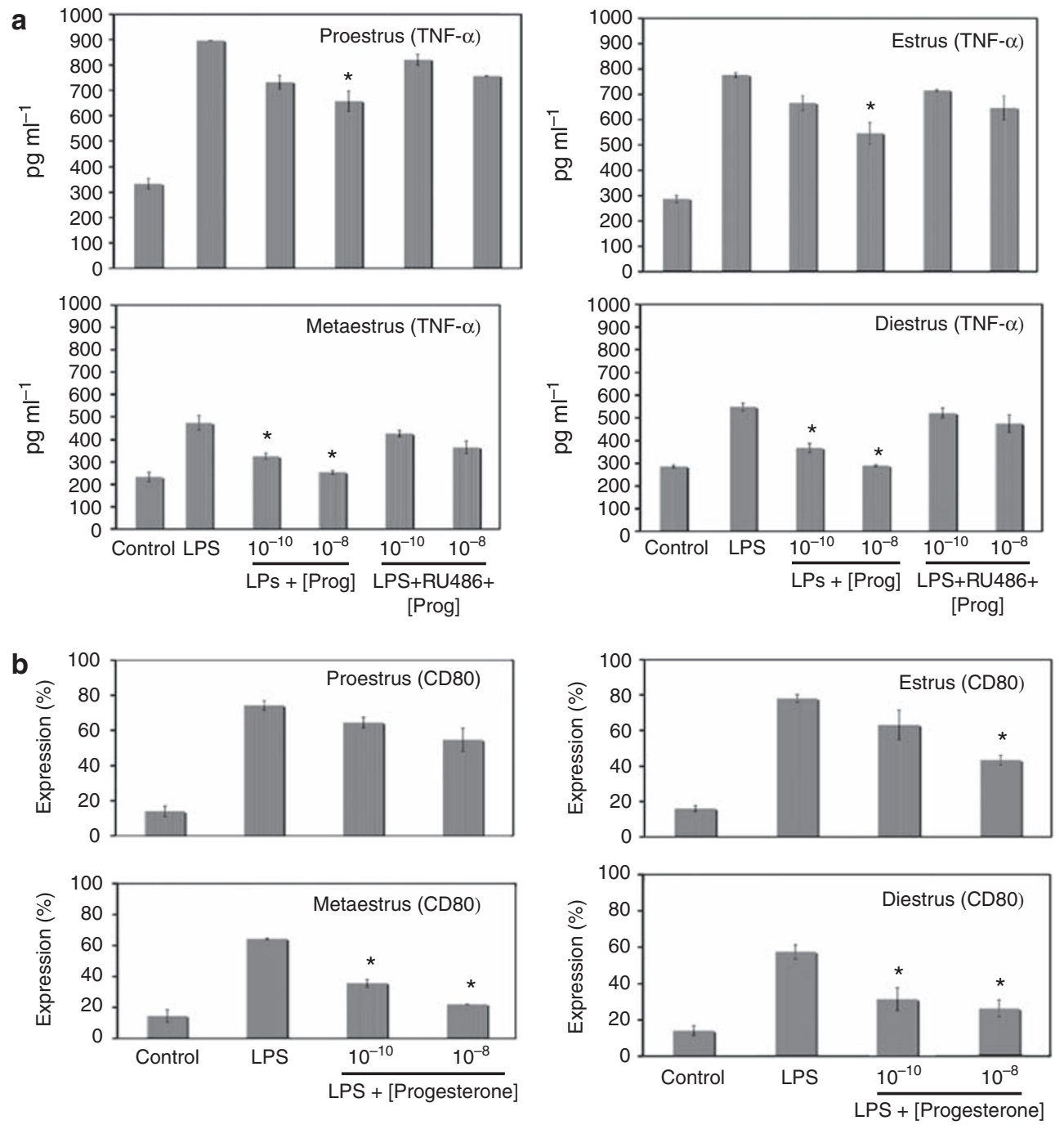

Figure 4 Progesterone effects differ throughout estrous cycle. Tumor necrosis factor- $\alpha$ (TNF- $\alpha$ ) secretion (a) and CD80 expression (b) by lipopolysaccharide (LPS)-stimulated splenic dendritic cells (DCs) from mice separated into stages of the estrous cycle associated with low (proestrus, estrus) and high (metaestrus, diestrus) progesterone levels were measured after pretreatment with progesterone and LPS stimulation. No treatment or ethanol alone served as controls. Mean \pm s.d. ${ }^{*}=P<0.05$ ( $n=6$ mice per estrous cycle stage).

of steroid hormone receptors in mediating these actions. To evaluate the mechanisms by which progesterone could mediate changes in DC activity, we examined phosphorylation and activation of the transcription factor STAT1. Phosphorylated STAT1 quickly diminished (within $20 \mathrm{~min}$ ) in the presence of progesterone, indicating a nongenomic mechanism of action. This is similar to previously reported nongenomic effects of steroid hormones on endometrial stromal cells. ${ }^{62,63}$

Steroid hormone levels fluctuate throughout the ovulatory cycle of all mammalian females, including humans and mice. This could result in differential regulation of immune responses by steroid hormones, depending on stage of the menstrual or estrous cycle. We show that PR expression by DCs fluctuates throughout the estrous cycle and that a greater number of DCs expressed PR in stages associated with higher progesterone levels (metaestrus, diestrus). DCs from mice in these stages also secreted lower levels of TNF- $\alpha$ and were less likely to express CD80 in response to LPS. In addition, DCs from mice in metae- strus and diestrus showed greater sensitivity to progesterone treatment, as evidenced by a more dramatic decline in cytokine production with increasing progesterone.

Similar to splenic DCs, uterine DC function was modified by progesterone. TNF- $\alpha$ production by uterine DCs was inhibited with progesterone treatment, especially by DCs from mice in diestrus. Moreover, these effects were shown in vivo. CD80 expression by splenic and lymph node DCs exposed to LPS IVAG was higher with mice in estrus, whereas fewer splenic and lymph node DCs from mice in diestrus expressed CD80 after LPS IVAG administration. Our findings are inconsistent with a report by Wieser et al..$^{40}$ that showed an increase in Langerhans cells in vaginal epithelium of women in response to progesterone. However, we observed an increase in leukocyte infiltration in vaginal swabs of animals in metaestrus and diestrus. Therefore, it is possible that the majority of DCs migrated to the vaginal epithelium of mice during metaestrus and diestrus (consistent with fewer uterine DCs identified at these stages). Our results are also in contrast 

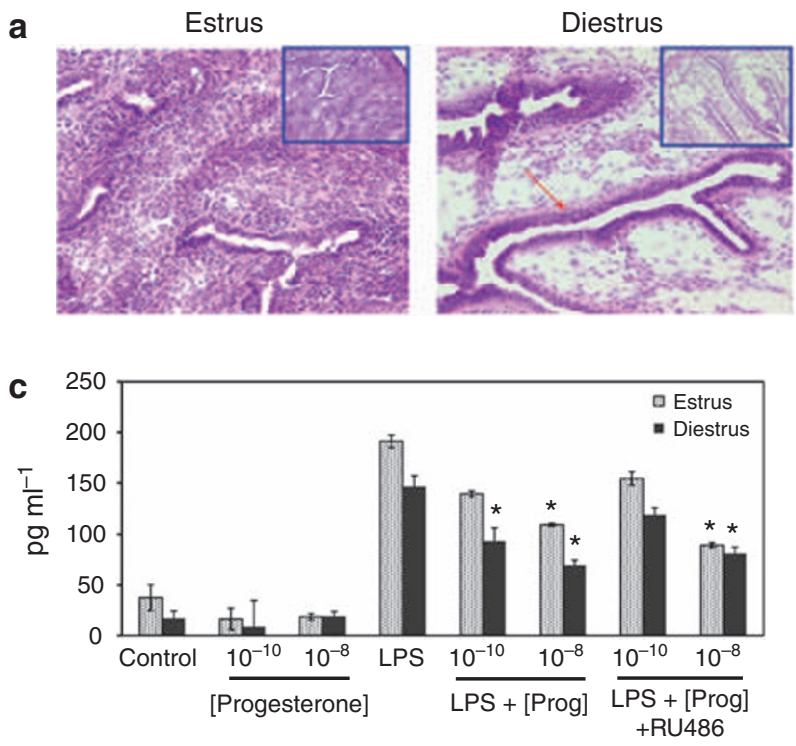
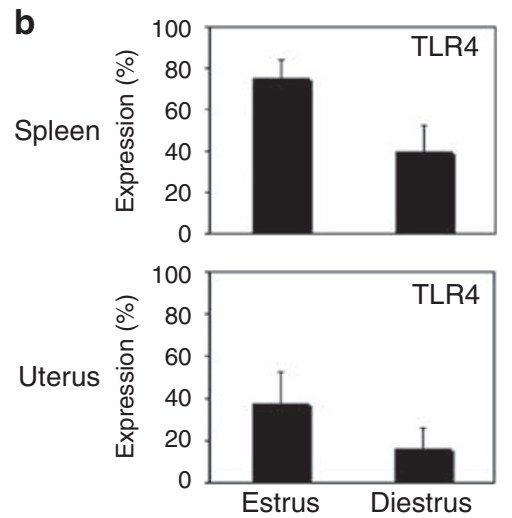

Figure 5 Progesterone effects in uterine tissue. Mice were separated into stages of the estrous cycle associated with low (estrus) and high (diestrus) progesterone levels to assess CD11 ${ }^{+}$dendritic cell (DC) function. Micrographs ( $\times 20$ larger, $\times 10$ inset) show differences in uterine tissue morphology at different stages and are representative of three independent experiments. Glandular epithelial cells are indicated by red arrows (a). (b) Toll-like receptor 4 (TLR4) expression by splenic and uterine DCs was evaluated ( $n=4$ mice per estrous cycle stage). (c) Tumor necrosis factor- $\alpha$ (TNF- $\alpha$ ) secretion by uterine DCs from mice separated into stages of the estrous cycle associated with low (estrus) and high (diestrus) progesterone levels were measured after pretreatment with progesterone and lipopolysaccharide (LPS) stimulation. Mean \pm s.d. $\left(n=4\right.$ mice per estrous cycle stage). ${ }^{*} P<0.05$.

a
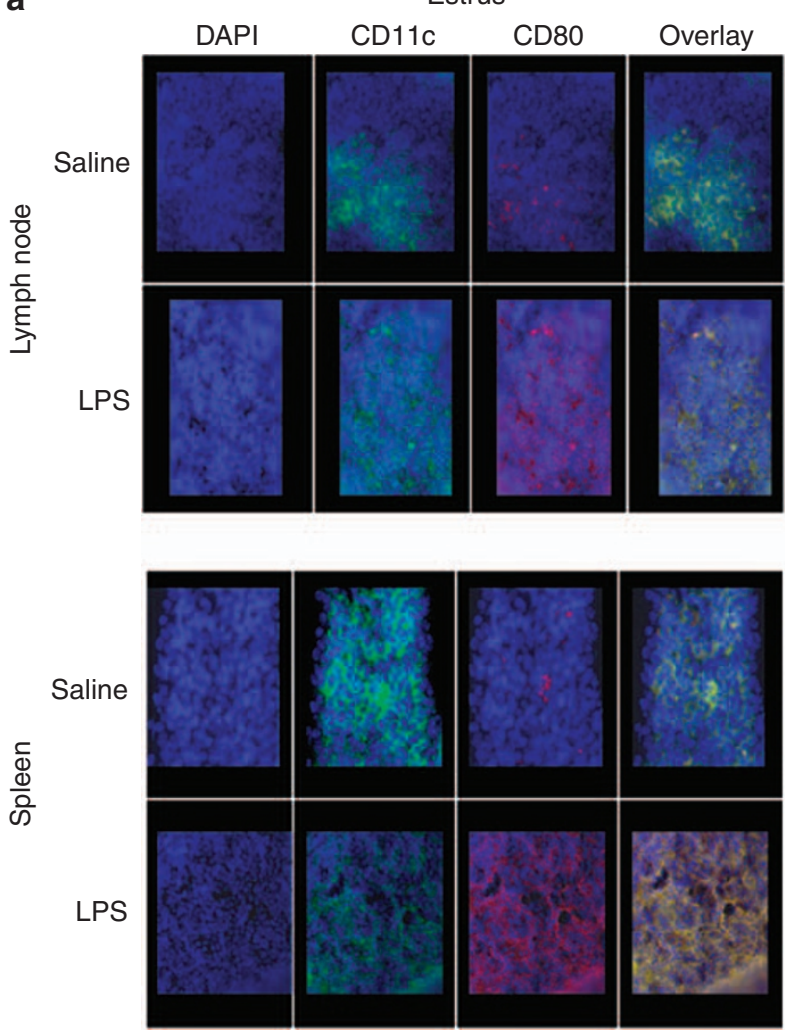

b
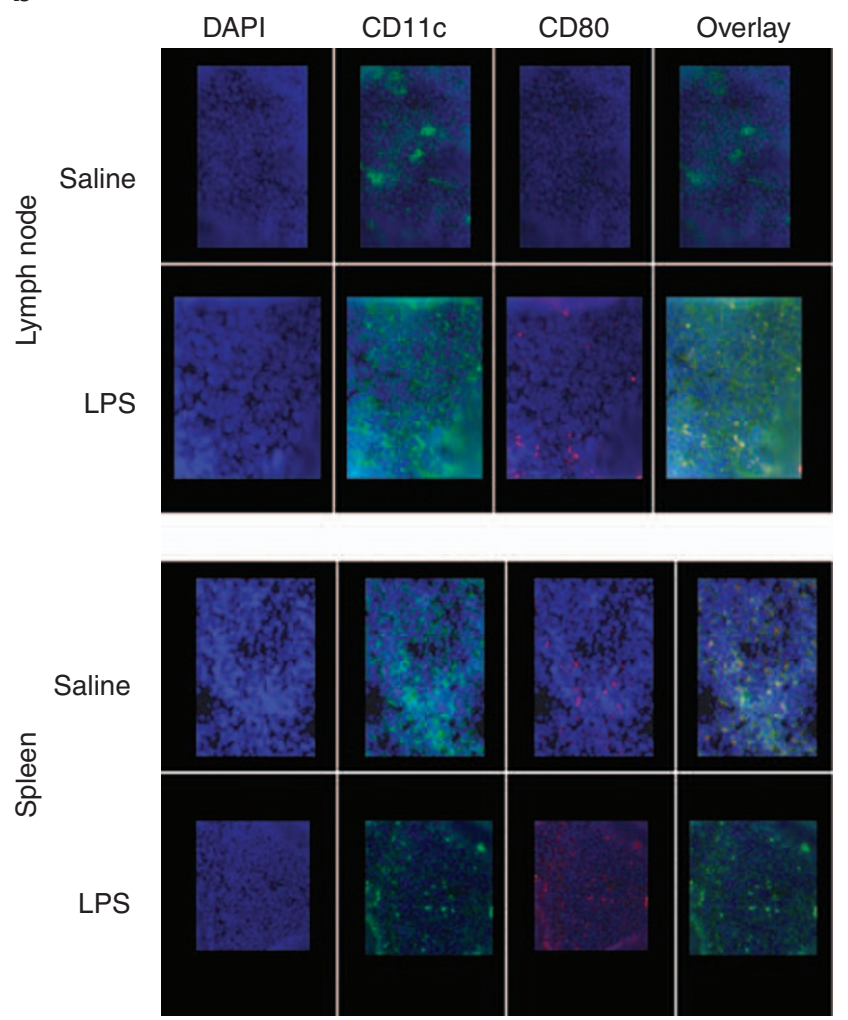

Figure 6 Effects of intravaginal administration of lipopolysaccharide (LPS) on dendritic cells (DCs) in mice at specific estrus cycle stages. Animals were separated into stages of the estrus cycle associated with low (estrus, a) and high (diestrus, b) progesterone levels and given LPS by intravaginal administration. CD11 $\mathrm{c}^{+}$DCs (green) were identified in lymph node and splenic tissues and examined for expression of the costimulatory molecule CD80 (red). DAPI (4'-6-Diamidino-2-phenylindole) labels cellular nuclei. Micrographs are representatative of four independent experiments. 
to a report by Yang et al. ${ }^{64}$ showing that progesterone can induce DC maturation. Possible factors contributing to this discrepancy include the specific functions analyzed. Their experiments focused on cytokine production by immature DCs, whereas our studies focused on cytokines produced by LPS-stimulated DCs. Our previous studies looking at immature rat DCs showed that progesterone could increase antigen uptake by immature DCs, but only at very high (pharmacological) concentrations. ${ }^{50}$

Some women have impaired immune responses to infectious agents and are more susceptible to genital tract infections compared with men (that is, greater susceptibility of pregnant women to H1N1 infection ${ }^{65}$ and increased rate of HIV infection ${ }^{66}$ ), signifying a role for sex hormones in regulating immunity. ${ }^{1,14,67}$ Our findings suggest that progesterone could have a role in regulating DC function and modifying immunity to pathogens in hormoneresponsive mucosal tissues, but that this is dependent on stage of the cycle and expression of the receptor for progesterone by DCs. During stages with low progesterone levels, females could mount strong immune responses, but be more susceptible to overstimulation of the immune system, which could lead to tissue damage and autoimmune/inflammatory conditions. During stages with higher progesterone levels, females could have difficulties generating a sufficient immune response and might be more susceptible to infections, thereby allowing pathogens to thrive in the host. Therefore, it is important to take into consideration the level and effects of steroid hormones such as progesterone in developing immune-based therapeutics for women.

\section{METHODS}

Animals. Female C57BL/6 mice were purchased from Taconic (Hudson, NY). Animals were housed under conditions of 12-h day/ night cycle and provided with food and water ad libitum. Animals were maintained in pathogen-free facilities, and all procedures were performed using approved protocols in accordance with the National Institute of Mental Health/NIH Animal Care and Use Committee.

Reagents. Progesterone, FITC-conjugated dextran, propidium iodide, LPS from Escherichia coli, and RU486 (mifepristone) were purchased from Sigma (St Louis, MO).

Antibodies. Purified antibodies that recognize and bind to amino acid residues 533-547 of the mouse PR $\left(100 \mu \mathrm{g} \mathrm{ml}^{-1}\right.$; diluted 1:5000) were purchased from Affinity Bioreagents (Golden, CO). Phycoerythrin (PE)conjugated antibodies to mouse CD80 $\left(0.1 \mathrm{mg} \mathrm{ml}^{-1}\right)$, PE-conjugated antibodies to TLR4 $\left(0.1 \mathrm{mg} \mathrm{ml}^{-1}\right), \mathrm{PE}$-conjugated antibodies to I-A $\left(0.1 \mathrm{mg} \mathrm{ml}^{-1}\right)$, FITC-conjugated goat anti-mouse antibody $\left(0.1 \mathrm{mg} \mathrm{ml}^{-1}\right)$, and appropriate isotype control antibodies were purchased from $\mathrm{BD}$ Biosciences (San Diego, CA). FITC-conjugated or PE-conjugated antibodies to mouse CD11c $\left(0.2 \mathrm{mg} \mathrm{ml}^{-1}\right)$ and appropriate isotype control antibodies were obtained from eBioscience (San Diego, CA).

Isolation of uterine and spleen DCs. Animals were killed to obtain uterine and spleen tissue collected in RPMI 1640 (Mediatech, Herndon, VA) containing 10\% charcoal-stripped serum (Biomeda; Foster City, CA), and $2 \% \mathrm{~L}$-glutamine and $2 \%$ penicillin-streptomycin (both from Sigma). Charcoal-stripped serum was used as a replacement for fetal calf serum as some components of serum have been shown to demonstrate hormonemimicking properties. Red blood cells of uterine and spleen tissue were removed using ACK lysis buffer (BioWhittaker; Walkersville, MD) for $10 \mathrm{~min}$ at $37^{\circ} \mathrm{C}$. To obtain DCs, single cell suspensions of splenocytes and uterine cells were labeled with magnetic bead-conjugated antibodies recognizing mouse $\mathrm{CD} 11 \mathrm{c}$, incubated at $4^{\circ} \mathrm{C}$ for $15 \mathrm{~min}$, and passed through magnetic columns using the Miltenyi Biotec (Auburn, CA) magnetic bead-based method to isolate CD11c ${ }^{+}$DCs.

Analysis of hormone receptor expression. Immature DCs (0.5$1.0 \times 10^{6}$ cells per tube) were collected into polystyrene Falcon tubes (BD Biosciences) and washed with fluorescence-activated cell sorter (FACS) buffer containing $1 \times$ phosphate-buffered saline (Molecular Biologicals, Columbia, MD), $2 \%$ charcoal-stripped serum (Biomeda), and $0.2 \%$ sodium azide (Sigma). Cells were centrifuged for $5 \mathrm{~min}$ at 2000 r.p.m. followed by supernatant removal to prepare for cell labeling. Cells were incubated with $10 \mu \mathrm{l}$ anti-mouse CD11c (eBioscience) and $10 \mu \mathrm{l}$ anti-mouse $\mathrm{I}-\mathrm{A}^{\mathrm{b}}$ (BD Pharmingen, San Diego, CA) on ice for $\sim 20$ min. Cells were washed with FACS buffer to remove excess antibody and centrifuged for $5 \mathrm{~min}$ at $2000 \mathrm{r}$ p.m. Supernatant was removed by decanting, and cells were treated with Cytofix/Cytoperm solution (BD Biosciences) for $20 \mathrm{~min}$ to permeabilize cells, followed by a washing step with Cytofix/Cytoperm wash buffer and centrifugation for $5 \mathrm{~min}$. Cells were incubated with $10 \mu \mathrm{l}$ mouse serum for $10 \mathrm{~min}$ to prevent nonspecific binding of antibodies to intracellular proteins. A volume of $10 \mu \mathrm{l}$ of antibodies to mouse PR (Affinity Bioreagents) or appropriate isotype control were added to tubes for $10 \mathrm{~min}$. Fluorochromeconjugated secondary antibody (goat anti-mouse) was added to each tube for an additional $10 \mathrm{~min}$. All incubations were carried out at $4^{\circ} \mathrm{C}$. Cells were collected into a BD FACSCalibur (BD Biosciences) and analyzed with FlowJo analysis software (Tree Star, Ashland, OR).

Hormone treatment and stimulation of uterine and splenic cultures. Cells $\left(\mathrm{CD} 11 \mathrm{c}^{+}\right)$isolated from spleen $\left(1.0 \times 10^{6}\right.$ cells $)$ or uterus $\left(2.5 \times 10^{5}\right.$ cells) were treated with progesterone dissolved in ethanol at indicated concentrations (added 1:100 in cultures). As fewer CD11 $\mathrm{c}^{+}$cells from uterine tissue were isolated from each animal (cells were not pooled to assess individual animal responses), smaller numbers of cells were used with uterine cell cultures. Splenic DCs and uterine DCs were cultured in $1 \mathrm{ml}$ total volume in 24-well plate or $0.25 \mathrm{ml}$ total volume in 96-well plate, respectively, to account for differences in cell numbers. After 2-h hormone pretreatment, LPS from E. coli $\left(1 \mu \mathrm{g} \mathrm{ml}^{-1}\right)$ was added to cultures. Cells untreated, treated with ethanol only, or treated with LPS or hormone alone served as experimental controls. Ethanol treatment alone increased DC cytokine production, but this was not statistically significant when compared with untreated cultures. Some LPS-stimulated cultures received the PR antagonist RU486 $\left(10^{-8} \mathrm{M}\right)$. Cells treated with RU486 alone served as additional control. Cultures remained incubated up to $48 \mathrm{~h}$ at $37^{\circ} \mathrm{C} / 5 \% \mathrm{CO}_{2}$.

Cytokine analysis. After treatment with progesterone, RU486, LPS, or a combination for $48 \mathrm{~h}$, supernatants were collected from cultured cells. TNF- $\alpha$, IL-1 $\beta$, and IL-12 secretion from cultured cells was determined using Searchlight multiplex array analysis service provided by Pierce Biotechnology (Woburn, MA).

Western blotting. Cells were collected, centrifuged, and resuspended in ice-cold RIPA buffer (50 mM Tris, $150 \mathrm{mM} \mathrm{NaCl}, 1 \%$ IgePal-630, $0.5 \%$ deoxycholate, $1 \mathrm{mM}$ EDTA) containing protease inhibitor mixture (Calbiochem, San Diego ,CA), $1 \mathrm{mM} \mathrm{NaF}$, and $1 \mathrm{mM}$ sodium orthovanadate. Samples were immediately frozen using dry ice, thawed, and then centrifuged. Whole cell supernatants were collected and stored at $-80^{\circ} \mathrm{C}$. Samples were run on NuPAGE 4-12\% Bis-Tris gels in MOPS SDS running buffer. Proteins were transferred to polyvinylidene difluoride membranes and blocked for $1 \mathrm{~h}$ in TBS containing $0.1 \%$ Tween 20 (TBST) with $5 \%$ nonfat dry milk for total STAT1 or $5 \%$ bovine serum albumin for phosphospecific STAT1. Membranes were then incubated in TBST containing $1 \%$ bovine serum albumin with mouse monoclonal antibodies (Invitrogen, Carlsbad, CA) specific for STAT1 $\alpha$ or phosphorylated STAT $1 \alpha / \beta$ (pY701) followed by horseradish peroxidase-conjugated secondary antibody (Santa Cruz Biotechnology, Santa Cruz, CA). Signal was 
detected on HyperECL film with the ECL Plus chemiluminescence kit (GE Healthcare, Piscataway, NJ). Densitometry analysis was performed using Image J software (National Institutes of Health, Bethesda, MD).

Vaginal smear collection. Vaginal smears from female mice were acquired daily for 3-4 weeks to determine regularity of estrous cycles. Vaginal secretions were collected aseptically using a smooth polished glass dropper, which was sanitized using $70 \%$ ethanol and rinsed with normal saline before collection of each sample. Each dropper was filled with 100 $150 \mu \mathrm{l}$ of sterile normal saline ( $\mathrm{NaCl} 0.9 \%)$ and tip gently inserted into the vaginal opening, $\sim 1 \mathrm{~mm}$. After insertion, fluid was expelled into the vagina approximately two to three times before collection of sample.

Estrous cycle stage determination. Vaginal secretions collected from mice $(\sim 75-100 \mu \mathrm{l})$ were placed on a microscope slide for subsequent evaluation. Vaginal smear cytology was used for detecting specific stage of estrous cycle. Proestrus typically occurs for $\sim 12 \mathrm{~h}$; estrus happens between 9 and $27 \mathrm{~h}$; metaestrus occurs for $6-8 \mathrm{~h}$; and diestrus will take place between 55 and $70 \mathrm{~h}$. Unstained material was observed under a light microscope to determine estrus cycle stage based on proportion among three cell types: round, nucleated epithelial cells; irregular-shaped, anucleated cornified cells; and small, round leukocytes. Proportion of each cell type was used to determine estrous cycle stage, as previously reported. ${ }^{68}$

Intravaginal administration. Mice were separated into specific stages of the estrous cycle and given $20 \mu \mathrm{LPS}\left(5 \mu \mathrm{gg}^{-1}\right)$ or FITC-dextran $\left(5 \mu \mathrm{gg}^{-1}\right)$ administered intravaginally. Mice receiving saline $(\mathrm{NaCl}$, $0.9 \%)$ served as a control. Mice were held in an inverted position for at least $5 \mathrm{~min}$ to ensure that the solution was absorbed into the vaginal cavity. Uterine, splenic, and lymph node tissues were collected $24 \mathrm{~h}$ after administration of proteins to assess any differences.

Tissue preparation for immunohistochemistry. Tissues (spleen, uterus, and lymph node) were fixed in Jung Tissue Freezing Medium (Leica Instruments, Nussloch, Germany) and stored at $-80^{\circ} \mathrm{C}$ until ready for sectioning. Tissue sections were prepared using Leica CM3050-Cryostat (Leica Microsystems). Cryostat was precooled at least $2 \mathrm{~h}$ before tissue sectioning to $-20^{\circ} \mathrm{C}$ (chamber temperature) and $-15^{\circ} \mathrm{C}$ (object stage temperature). Excess freezing media was trimmed from specimens to yield a $3 \mathrm{~mm} \times 3 \mathrm{~mm}$ frozen square of tissue specimen. Using Leica disposable microtome blades (Leica Instruments), tissues were sliced at $5-10 \mu \mathrm{m}$ sections and fixed to glass microscope slides with $4 \%$ paraformaldehyde for a minimum of $10 \mathrm{~min}$. Slides were stored at $-20^{\circ} \mathrm{C}$ until ready for hematoxylin and eosin or immunohistochemical staining.

Statistical analysis. For all statistical analysis, the level of significance was set at a probability of no more than 0.05 to be considered significant. Data are presented as mean values \pm standard deviation. Statistical analysis was performed using analysis of variance (ANOVA) and Student's $t$-test comparing treatments with LPS.

SUPPLEMENTARY MATERIAL is linked to the online version of the paper at http://www.nature.com/mi

\section{ACKNOWLEDGMENTS}

This work was supported by the Intramural Research Program of the National Institute of Mental Health (NIMH)/NIH and by a biodefense grant from the National Institute of Allergy \& Infectious Diseases (NIAID)/NIH Intramural Research Program. We thank Dr Cecilia Tami for careful manuscript review and technical assistance.

\section{DISCLOSURE}

The authors declared no conflict of interest.

\section{REFERENCES}

1. Mingjia, L. \& Short, R. How oestrogen or progesterone might change a woman's susceptibility to HIV-1 infection. Aust. N.Z. J. Obstet. Gynaecol. 42, 472-475 (2002).

2. Kaushic, C., Ashkar, A.A., Reid, L.A. \& Rosenthal, K.L. Progesterone increases susceptibility and decreases immune responses to genital herpes infection. J. Virol. 77, 4558-4565 (2003).

3. Kaushic, C. The role of the local microenvironment in regulating susceptibility and immune responses to sexually transmitted viruses in the female genital tract. J. Reprod. Immunol. 83, 168-172 (2009).

4. Dayan, L. \& Donovan, B. Chlamydia, gonorrhoea, and injectable progesterone. Lancet 364, 1387-1388 (2004).

5. Fahey, J.V. et al. Estradiol selectively regulates innate immune function by polarized human uterine epithelial cells in culture. Mucosal Immunol. 1, 317-325 (2008).

6. Shust, G.F. et al. Female genital tract secretions inhibit herpes simplex virus infection: correlation with soluble mucosal immune mediators and impact of hormonal contraception. Am. J. Reprod. Immunol. 63, 110-119 (2010).

7. Bombail, V., MacPherson, S., Critchley, H.O. \& Saunders, P.T. Estrogen receptor related beta is expressed in human endometrium throughout the normal menstrual cycle. Hum. Reprod. 23, 2782-2790 (2008).

8. Witkin, S.S., Linhares, I.M. \& Giraldo, P. Bacterial flora of the female genital tract: function and immune regulation. Best. Pract. Res. Clin. Obstet. Gynaecol. 21, 347-354 (2007).

9. Iwasaki, A. Mucosal dendritic cells. Annu. Rev. Immunol. 25, 381-418 (2007).

10. Raz, E. Mucosal immunity: aliment and ailments. Mucosal Immunol. 3, 4-7 (2010).

11. Shen, R. et al. Macrophages in vaginal but not intestinal mucosa are monocyte-like and permissive to human immunodeficiency virus type 1 infection. J. Virol. 83, 3258-3267 (2009).

12. Nikolic, T., Geutskens, S.B., van Rooijen, N., Drexhage, H.A. \& Leenen, P.J. Dendritic cells and macrophages are essential for the retention of lymphocytes in (peri)-insulitis of the nonobese diabetic mouse: a phagocyte depletion study. Lab. Invest. 85, 487-501 (2005).

13. Healey, G.D., Elvin, S.J., Morton, M. \& Williamson, E.D. Humoral and cellmediated adaptive immune responses are required for protection against Burkholderia pseudomallei challenge and bacterial clearance postinfection. Infect. Immun. 73, 5945-5951 (2005)

14. Nehete, P.N. et al. Protection by dendritic cells-based HIV synthetic peptide cocktail vaccine: preclinical studies in the SHIV-rhesus model. Vaccine 23, 2154-2159 (2005).

15. Pulendran, B. Variegation of the immune response with dendritic cells and pathogen recognition receptors. J. Immunol. 174, 2457-2465 (2005).

16. Kelsall, B.L., Biron, C.A., Sharma, O. \& Kaye, P.M. Dendritic cells at the host-pathogen interface. Nat. Immunol. 3, 699-702 (2002).

17. Zhang, Z. et al. The characteristics of tolerogenic plasmacytoid dendritic cells stimulated with interleukin-3. Transplant. Proc. 37, 7-9 (2005).

18. Jiang, $\mathrm{H}$. et al. Administration of tolerogenic dendritic cells induced by interleukin-10 prolongs rat splenic allograft survival. Transplant. Proc. 36, 3255-3259 (2004)

19. Riboldi, E. et al. Cutting edge: proangiogenic properties of alternatively activated dendritic cells. J. Immunol. 175, 2788-2792 (2005).

20. Zeyda, M. et al. Polyunsaturated fatty acids block dendritic cell activation and function independently of NF-kappaB activation. J. Biol. Chem. 280, 14293-14301 (2005).

21. $\mathrm{Xu}, \mathrm{H}$. et al. IL-12 p35 silenced dendritic cells modulate immune responses by blocking IL-12 signaling through JAK-STAT pathway in T lymphocytes. Biochem. Biophys. Res. Commun. 353, 812-816 (2007).

22. Wenta, N., Strauss, H., Meyer, S. \& Vinkemeier, U. Tyrosine phosphorylation regulates the partitioning of STAT1 between different dimer conformations. Proc. Natl. Acad. Sci. USA 105, 9238-9243 (2008).

23. Johnson, L.M. \& Scott, P. STAT1 expression in dendritic cells, but not T cells, is required for immunity to Leishmania major. J. Immunol. 178, 7259-7266 (2007)

24. Kalady, M.F. et al. Sequential delivery of maturation stimuli increases human dendritic cell IL-12 production and enhances tumor antigenspecific immunogenicity. J. Surg. Res. 116, 24-31 (2004).

25. Moser, M. \& Murphy, K.M. Dendritic cell regulation of TH1-TH2 development. Nat. Immunol. 1, 199-205 (2000).

26. Zhou, L.J. \& Tedder, T.F. A distinct pattern of cytokine gene expression by human CD83+ blood dendritic cells. Blood 86, 3295-3301 (1995). 
27. Kelsall, B.L., Stuber, E., Neurath, M. \& Strober, W. Interleukin-12 production by dendritic cells. The role of CD40-CD40L interactions in Th1 T-cell responses. Ann. NY Acad. Sci. 795, 116-126 (1996).

28. Gilliet, M. et al. The development of murine plasmacytoid dendritic cell precursors is differentially regulated by FLT3-ligand and granulocyte/ macrophage colony-stimulating factor. J. Exp. Med. 195, 953-958 (2002).

29. Colonna, M., Pulendran, B. \& Iwasaki, A. Dendritic cells at the hostpathogen interface. Nat. Immunol. 7, 117-120 (2006).

30. Collins, M.K., Tay, C.S. \& Erlebacher, A. Dendritic cell entrapment within the pregnant uterus inhibits immune surveillance of the maternal/fetal interface in mice. J. Clin. Invest. 119, 2062-2073 (2009).

31. Keenihan, S.N. \& Robertson, S.A. Diversity in phenotype and steroid hormone dependence in dendritic cells and macrophages in the mouse uterus. Biol. Reprod. 70, 1562-1572 (2004).

32. Graziano, V., Check, J.H., Dietterich, C., Choe, J.K. \& Yuan, W. A comparison of luteal phase support in graduated estradiol/progesterone replacement cycles using intramuscular progesterone alone versus combination with vaginal suppositories on outcome following frozen embryo transfer. Clin. Exp. Obstet. Gynecol. 32, 93-94 (2005).

33. Mizutani, T. et al. Effect of steroid add-back therapy on the proliferative activity of uterine leiomyoma cells under gonadotropin-releasing hormone agonist therapy. Gynecol. Endocrinol. 20, 80-83 (2005).

34. Lydon, J.P. et al. Mice lacking progesterone receptor exhibit pleiotropic reproductive abnormalities. Genes Dev. 9, 2266-2278 (1995).

35. Ismail, P.M. et al. Progesterone involvement in breast development and tumorigenesis--as revealed by progesterone receptor "knockout" and "knockin" mouse models. Steroids 68, 779-787 (2003).

36. Kaushic, C., Murdin, A.D., Underdown, B.J. \& Wira, C.R. Chlamydia trachomatis infection in the female reproductive tract of the rat: influence of progesterone on infectivity and immune response. Infect. Immun. 66, 893-898 (1998)

37. Stites, D.P. \& Siiteri, P.K. Steroids as immunosuppressants in pregnancy. Immunol. Rev. 75, 117-138 (1983)

38. Miyaura, H. \& Iwata, M. Direct and indirect inhibition of Th1 development by progesterone and glucocorticoids. J. Immunol. 168, 1087-1094 (2002)

39. Sentman, C.L., Meadows, S.K., Wira, C.R. \& Eriksson, M. Recruitment of uterine NK cells: induction of CXC chemokine ligands 10 and 11 in human endometrium by estradiol and progesterone. J. Immunol. 173, 6760-6766 (2004).

40. Wieser, F. et al. Progesterone increases the number of Langerhans cells in human vaginal epithelium. Fertil Steril. 75, 1234-1235 (2001).

41. Hel, Z., Stringer, E. \& Mestecky, J. Sex steroid hormones, hormonal contraception, and the immunobiology of human immunodeficiency virus-1 infection. Endocr. Rev. 31, 79-97 (2010).

42. Hepworth, M.R., Hardman, M.J. \& Grencis, R.K. The role of sex hormones in the development of Th2 immunity in a gender-biased model of Trichuris muris infection. Eur. J. Immunol. 40, 406-416 (2010).

43. Segerer, S.E. et al. Impact of female sex hormones on the maturation and function of human dendritic cells. Am. J. Reprod. Immunol. 62, 165-173 (2009).

44. Yang, L., Hu, Y. \& Hou, Y. Effects of 17 beta-estradiol on the maturation, nuclear factor kappa B p65 and functions of murine spleen CD11cpositive dendritic cells. Mol. Immunol. 43, 357-366 (2006).

45. Cutolo, M. et al. Sex hormones modulate the effects of Leflunomide on cytokine production by cultures of differentiated monocyte/macrophages and synovial macrophages from rheumatoid arthritis patients. J. Autoimmun. 32, 254-260 (2009).

46. Prieto, G.A. \& Rosenstein, Y. Oestradiol potentiates the suppressive function of human CD4 CD25 regulatory T cells by promoting their proliferation. Immunology 118, 58-65 (2006).

47. Islander, U. et al. Estren-mediated inhibition of T Iymphopoiesis is estrogen receptor-independent whereas its suppression of T cellmediated inflammation is estrogen receptor-dependent. Clin. Exp. Immunol. 139, 210-215 (2005).
48. Kovats, S. \& Carreras, E. Regulation of dendritic cell differentiation and function by estrogen receptor ligands. Cell Immunol. 252, 81-90 (2008).

49. Bird, M.D., Karavitis, J. \& Kovacs, E.J. Sex differences and estrogen modulation of the cellular immune response after injury. Cell Immunol. 252, 57-67 (2008).

50. Butts, C.L. et al. Progesterone inhibits mature rat dendritic cells in a receptor-mediated fashion. Int. Immunol. 19, 287-296 (2007).

51. Ivanova, E. et al. CD83 monocyte-derived dendritic cells are present in human decidua and progesterone induces their differentiation in vitro. Am J. Reprod. Immunol. 53, 199-205 (2005).

52. Hughes, G.C., Thomas, S., Li, C., Kaja, M.K. \& Clark, E.A. Cutting edge: progesterone regulates IFN-alpha production by plasmacytoid dendritic cells. J. Immunol. 180, 2029-2033 (2008).

53. Kawana, K., Kawana, Y. \& Schust, D.J. Female steroid hormones use signal transducers and activators of transcription protein-mediated pathways to modulate the expression of T-bet in epithelial cells: a mechanism for local immune regulation in the human reproductive tract. Mol. Endocrinol. 19, 2047-2059 (2005).

54. lijima, N., Thompson, J.M. \& Iwasaki, A. Dendritic cells and macrophages in the genitourinary tract. Mucosal. Immunol. 1, 451-459 (2008).

55. Vakkila, J., DeMarco, R.A. \& Lotze, M.T. Imaging analysis of STAT1 and NF-kappaB translocation in dendritic cells at the single cell level. J. Immunol. Methods 294, 123-134 (2004).

56. Benco, A. et al. Involvement of the transcription factor STAT1 in the regulation of porcine ovarian granulosa cell functions treated and not treated with ghrelin. Reproduction 138, 553-560 (2009).

57. Zoumpoulidou, G. et al. Convergence of interferon-gamma and progesterone signaling pathways in human endometrium: role of PIASy (protein inhibitor of activated signal transducer and activator of transcription-y). Mol. Endocrinol. 18, 1988-1999 (2004).

58. Ardavin, C. et al. Origin and differentiation of dendritic cells. Trends Immunol. 22, 691-700 (2001).

59. Chen, M. et al. Dendritic cell apoptosis in the maintenance of immune tolerance. Science 311, 1160-1164 (2006).

60. Thongngarm, T., Jenkins, J.K., Ndebele, K. \& McMurray, R.W. Estrogen and progesterone modulate monocyte cell cycle progression and apoptosis. Am. J. Reprod. Immunol. 49, 129-138 (2003).

61. Tibbetts, T.A., Conneely, O.M. \& O'Malley, B.W. Progesterone via its receptor antagonizes the pro-inflammatory activity of estrogen in the mouse uterus. Biol. Reprod. 60, 1158-1165 (1999).

62. Bruscoli, S. et al. Genomic and non-genomic effects of different glucocorticoids on mouse thymocyte apoptosis. Eur. J. Pharmacol. 529, 63-70 (2006)

63. Vallejo, G., Ballare, C., Baranao, J.L., Beato, M. \& Saragueta, P. Progestin activation of non-genomic pathways via crosstalk of $P R$ with $E R \backslash\{b e t a \backslash\}$ induces proliferation of endometrial stromal cells. Mol. Endocrinol. 19, 3023-3037 (2005)

64. Yang, L., Li, X., Zhao, J. \& Hou, Y. Progesterone is involved in the maturation of murine spleen CD11c-positive dendritic cells. Steroids 71, 922-929 (2006).

65. Jamieson, D.J. et al. H1N1 2009 influenza virus infection during pregnancy in the USA. Lancet 374, 451-458 (2009).

66. Lund, J.M., Linehan, M.M., lijima, N. \& Iwasaki, A. Cutting Edge: plasmacytoid dendritic cells provide innate immune protection against mucosal viral infection in situ. J. Immunol. 177, 7510-7514 (2006).

67. Enomoto, L.M., Kloberdanz, K.J., Mack, D.G., Elizabeth, D. \& Weinberg, A. Ex vivo effect of estrogen and progesterone compared with dexamethasone on cell-mediated immunity of HIV-infected and uninfected subjects. J. Acquir. Immune. Defic. Syndr. 45, 137-143 (2007).

68. Marcondes, F.K., Bianchi, F.J. \& Tanno, A.P. Determination of the estrous cycle phases of rats: some helpful considerations. Braz. J. Biol. 62, 609614 (2002). 